\title{
Pain measurement in research and practice
}

\author{
Kurt Kroenke, $M D^{1,2}$ \\ 'VA HSR\&D Center for Health Communication and Information, Regenstrief Institute, Inc., Indianapolis, IN, USA; ${ }^{2}$ Indiana University School of \\ Medicine, Indianapolis, IN, USA.
}

J Gen Intern Med 33(Suppl 1):S7-S8 DOI: $10.1007 / \mathrm{s} 11606-018-4363-4$

๑) Society of General Internal Medicine 2018

$\mathrm{P}$ ain is among the most prevalent, persistent, and costly health conditions in clinical practice as well as the general population. ${ }^{1}$ Moreover, musculoskeletal pain conditions account for four of the nine most disabling diseases. ${ }^{2}$ Unlike medical disorders such as diabetes and hypertension which can be evaluated and monitored with tests or devices independent of the patient, pain is reliant on patient report to determine its severity as well as response to treatment. Patient-reported outcomes (PROs) are central to assessing symptoms, functional status, and other domains of health-related quality of life. Measurement-based care using PROs has proven an essential component of improving depression outcomes and has a similar salience for pain. Ideally, pain and other PRO measures used in research would not differ from those applied in patient care. After all, the metrics for $\mathrm{A} 1 \mathrm{C}$, blood pressure, and cholesterol are identical for investigators and clinicians, making the findings from clinical trials and other research easily interpretable and directly transferrable to clinical practice.

The Veterans Health Administration (VHA) Health Services Research and Development Service sponsored a state-ofthe-art research (SOTA) conference in November 2016 focused on nonpharmacological treatments for chronic musculoskeletal pain. Although numerous pain measures have been used in clinical research, there has been insufficient evidence to recommend one measure over another. Recognizing the advantages of using a common set of measures across studies, the SOTA suggested that a process be initiated to identify core measures for VA research. Therefore, the VHA convened a work group for which the principal charge was "to recommend core outcome measures for pain intensity and interference to be used in all VHA prospective clinical research studies of chronic musculoskeletal pain (both interventional and observational)." Musculoskeletal pain (e.g., low back and neck pain, arthritis, fibromyalgia, regional pain syndromes) represents $75-80 \%$ of all pain disorders in clinical practice and the majority of pain-related disability. Several streams of evidence have informed the work group's recommendations including a systematic review in this issue of the journal, ${ }^{3}$ an overview of

Published online April 9, 2018 recommendations formulated by other consensus groups, and the several Delphi surveys of work group members.

The systematic review, conducted by Goldsmith and colleagues, ${ }^{3}$ found for 17 different pain intensity and interference measures, only 43 articles that met the inclusion criteria which, in particular, required reporting at least one of four psychometric characteristics (minimally important difference, responsiveness, validity, test-retest reliability). While the relatively small number of articles covering so many measures may in part reflect the stringency of inclusion criteria, it indicates to a greater degree the fact that measures are often adopted by clinical researchers based upon one or a few psychometric studies. This in turn might be attributable to the paucity of funding for development and validation of scales; one prominent exception is the NIH investment of more than $\$ 100$ million dollars in the Patient-Reported Outcomes Measurement Information System (PROMIS) scales. Many other legacy scales have been developed and validated by individual investigators with either a minimal amount of funding or as secondary data analyses from larger studies funded for other reasons (e.g., clinical trials or cohort studies).

Goldsmith and colleagues found that five measures had data reported on all four psychometric criteria, and seven measures had data reported on three of the criteria. Based upon this review and other studies, the VHA measurement work group has concluded there is no clear psychometric winner among the pain measures for several reasons: (1) the review found only a small number of studies for any given measure; (2) there were substantial methodological and population differences across these studies; (3) studies comparing the same measures across different populations can show substantial psychometric differences $^{4}$; and (4) studies comparing different measures within the same population tend to show comparable responsiveness. ${ }^{5,6}$

Besides strong psychometrics, other characteristics considered important, particularly for uptake into clinical practice and also for research studies where many domains besides pain are being assessed, include the measure being brief, selfadministered, freely available (i.e., public domain), easy to score, widely accessible (e.g., downloadable from websites), and having actionable scores that can guide diagnostic or therapeutic decision-making. ${ }^{7}$ Three characteristics ranked highly in a Delphi survey of VHA work group members include the measure being brief, public domain, and feasible for clinical use including integration into the electronic health records. Notably, studies have shown that the performance of 
brief (less than ten items) or ultra-brief (two to four items) pain measures is similar enough to longer measures to justify the use of short measures in most clinical practice and many research settings. ${ }^{4-6}$ For example, a reliable and valid assessment of pain intensity and interference can be done with three items (PEG scale), five items (PROMIS four-item interference scale plus a single item numerical rating scale [NRS] assessing average pain in the past week), or eight items (Brief Pain Inventory seven-item interference scale plus the NRS item).

One debate in pain measurement is whether separate scores for pain intensity and interference are necessary or whether a composite measure combining the two domains into a single score is sufficient. A composite score is supported by recent research, ${ }^{5,6}$ which is helpful since a single score for either clinical decision-making or as a primary research outcome is desirable. The PEG which consists of one pain intensity and two pain interference items is one such composite measure that has been recommended by the US Surgeon General's Turning the Tide campaign to reduce opioid use and by the National Pain Strategy as an ultra-brief and public domain measure.

Other outcomes considered important in pain research by both the VHA measurement work group and other consensus panels ${ }^{8-10}$ include physical functioning and depression (ranked most highly) followed by sleep and anxiety. Although the VHA work group final report will propose a recommended measure for both pain intensity and interference as well as these secondary domains, there is emerging research on how to cross-walk scores among measures which will facilitate the comparison of results across studies that use different measures. ${ }^{4,11}$

The existence of valid pain and other PRO measures does not guarantee their use in clinical practice. Completion by interview and self-administration using paper forms followed by clinician scoring are relatively inefficient approaches. Computers and other technology can facilitate measurement in several ways. PRO administration and automated scoring using iPads or kiosks minimizes clinic personnel time. Web-based or interactive-voice response (IVR) phone administration enables patient completion of PROs at home before or after clinic visits. Embedding PRO scores into the electronic health record makes the information available at the point of care for clinical decision-making as well as longitudinal monitoring. The development of phone-based apps can foster more sophisticated pain tracking (e.g., ecological momentary assessment) as well as prompted self-management and treatment adherence. Pragmatic trials using collaborative or telecare models as well as vanguard clinical practices have used one or more of these strategies to enhance the feasibility and utility of PRO assessment.

Measurement is necessary but not sufficient for improving the care of patients with chronic pain. Pragmatic trials have shown that a stepped care approach using non-opioid analgesics sequentially or in combination can improve pain and reduce opioid use. Nonpharmacological approaches such as exercise, cognitive-behavioral therapy, yoga, acupuncture, chiropractic, and massage can be beneficial, although strategies to enhance patient adherence as well as policies to increase the accessibility to and payment for these treatments are needed. Similar to depression, studies in patients with chronic pain have shown that collaborative care or telecare models using nurse care managers are effective and efficient interventions; however, reimbursement policies need to be realigned in order to implement these evidence-based models. In short, measurement must be coupled with delivery of costeffective treatments in order to optimize pain outcomes.

Accurate and longitudinal measurement helps clinicians and patients work as partners to achieve target goals for diabetes, hypertension, hyperlipidemia, weight loss, and many other medical conditions. Tangible metrics provide a lingua franca for communicating with patients as well as with other clinicians who are co-managing a particular patient. Just as the numbers attached to A1C levels, blood pressure, cholesterol, and body weight all inform clinical decisions, the scores obtained from pain measures and other PROs can guide symptom management.

Corresponding Author: Kurt Kroenke, MD; VA HSR\&D Center for Health Communication and Information, Regenstrief Institute, Inc., Indianapolis, IN, USA (e-mail: kkroenke@regenstrief.org).

\section{Compliance with ethical standards:}

Conflict of interest: The author declares that he does not have a conflict of interest.

\section{REFERENCES}

1. Institute of Medicine. Relieving pain in America: a blueprint for transforming prevention, care, education, and research. Washington, DC: National Academies Press; 2011.

2. US Burden of Disease Collaborators. The state of US health, 1990-2010: burden of diseases, injuries, and risk factors. JAMA. 2013;310(6):591608.

3. Goldsmith ES, Taylor BC, Greer N, et al. Focused evidence review: psychometric properties of patient-reported outcome measures for chronic musculoskeletal pain. JGIM. 2018. https://doi.org/10.1007/ s11606-018-4327-8

4. Chen CX, Kroenke $\mathbf{K}$, Stump T, et al. Estimating minimally important differences for the PROMIS pain interference scales: results from three randomized clinical trials. Pain. 2018, (in press).

5. Krebs EE, Bair MJ, Wu J, Damush TM, Tu W, Kroenke K. Comparative responsiveness of pain outcome measures among primary care patients with musculoskeletal pain. Med Care 2010;48:1007-1014.

6. Kean J, Monahan PO, Kroenke $\mathbf{K}$, et al. Comparative responsiveness of the PROMIS pain interference short forms, brief pain inventory, PEG, and SF-36 bodily pain subscale. Med Care 2016;54(4):414-421.

7. Kroenke K, Monahan PO, Kean J. Pragmatic characteristics of patientreported outcome measures are important for use in clinical practice. J Clin Epidemiol 2015;68(9): 1085-1092.

8. Dworkin RH, Turk DC, Farrar JT, et al. Core outcome measures for chronic pain clinical trials: IMMPACT recommendations. Pain 2005;113(1-2):9-19.

9. Deyo RA, Dworkin SF, Amtmann D, et al. Report of the NIH Task Force on research standards for chronic low back pain. J Pain 2014;15(6):569585.

10. Chiarotto A, Terwee $\mathbf{C B}$, Ostelo RW. Choosing the right outcome measurement instruments for patients with low back pain. Best Pract Res Clin Rheumatol 2016;30(6):1003-1020.

11. Cook KF, Schalet BD, Kallen MA, Rutsohn JP, Cella D. Establishing a common metric for self-reported pain: linking BPI Pain Interference and SF-36 bodily pain subscale scores to the PROMIS Pain Interference metric. Qual Life Res 2015;24(10):2305-2318. 